\title{
The Mechanism and Treatment of Teenager Internet Addiction from Stress: A Review
}

\author{
Mengyuan Huang ${ }^{1, *}, \dagger$ and Weiyi Zhang ${ }^{2, \dagger}$ \\ ${ }^{1}$ Collge of foreign languages, Xinxiang Medical University, Xinxiang, China, 453000 \\ ${ }^{2}$ Collge of media, Nanjing University Jinling College, Nanjing, China, 210000 \\ ${ }^{*}$ Corresponding author. Email: 20191250026@stu.xxmu.edu.cn \\ These authors contributed equally.
}

\begin{abstract}
The phenomenon of Internet addiction among teenagers is becoming more and more serious with the increasing competition stress in contemporary society and the rapid development and popularization of the Internet. The health and social problems caused by Internet addiction have become one of the focuses of public attention. In this paper, we review and synthesize the extant studies to discuss the mechanism and treatment of teenagers' stress and internet addiction. According to the analysis of teenagers' Internet addiction behavior motivation to stress source, the author summarized the treatment methods and limitations of western medicine and traditional Chinese medicine for this disease in recent years, which can provide experience summary for teenagers to effectively prevent Internet addiction.
\end{abstract}

Keywords: stress, internet addiction, teenagers, mechanism of action, traditional Chinese medicine treatment, psychological prevention

\section{INTRODUCTION}

As an important medium with entertainment and social functions, the Internet has become an important means for teenagers to relieve their growing stress with the arrival of the $5 \mathrm{G}$ era. According to the 45 th statistical report on China's Internet Network Development released by China Internet Network Information Center (CNNIC) on April 28, 2020, by the end of March 2020, the number of Internet users in China had reached 904 million, and the Internet penetration rate had reached 64.5 percent, among which 26.9 percent were teenagers.

"Internet addiction Disorder" was first proposed by Ivan Goldberg in 1994 to describe the phenomenon of psychosocial damage caused by excessive Internet use. Young, described Internet addiction as a substance addiction disease like gambling, alcoholism, and drug abuse. Zhou focused on the psychological and physiological dependence of addicts on the Internet, and she believes that the cause of Internet addiction is a chronic periodic obsession state caused by repeated use of the Internet, resulting in an irresistible desire to use again [1]. From the perspective of users, Li highlights the harm of Internet addiction and describes it as a behavioral pattern that negatively affects users' academic performance, time management and sleep [2].
Teenagers are in a critical period of knowledge accumulation and personality development. Long-term addiction to the virtual world of the Internet will inevitably bring various negative impacts on their physical and mental health, academic development and interpersonal relations. In terms of physical and mental health, teenagers surfing the Internet for a long time will not only have health problems such as irregular schedules for work, rest and diet, decreased vision and cervical spondylosis, but also have negative emotions such as anxiety and depression. In terms of academic development, those with Internet addiction indulge in the virtual world of the Internet, and lose interest in learning, with the occurrence of being tired of learning and skipping classes. In terms of interpersonal relations, if teenagers indulge in the virtual world of the Internet for a long time, they will not only gradually lose the ability to interact with people in the real world, resulting in a sense of loneliness and loss, but also be easily induced by misconducts due to the lack of the supervision on interpersonal relations with parents and teachers as the core. Therefore, based on the universality and severity of Internet addiction among contemporary teenagers, this study aims to explore the mechanism of Internet addiction among college students, and then puts forward 
feasible suggestions for the treatment and prevention of Internet addiction among college students.

\section{THE MECHANISM OF STRESS AFFECTING INTERNET ADDICTION}

"Stress", as an environmental factor affecting addictive behavior [3], at the same time, has also been widely concerned by many scholars in the study of variables affecting teenagers' Internet addiction. Zhang found that college students' stressful life events are significantly positively correlated with internet addiction, and stressful life events can significantly predict Internet addiction [4].

In terms of the characteristics of the network itself, it can greatly meet the needs of individuals for autonomy, energy and relationship [5]. Therefore, teenagers tend to adopt negative coping methods in reality [6] when their basic psychological needs are not satisfied, their internal needs will be transformed into the motivation of game addiction $[5,7,8]$.

Davis' behavior-cognitive model describes the personal factors and environmental factors that make individuals produce "non-adaptive behaviors", which in turn promotes Internet addiction behaviors [33]. Based on the inspiration of this model, Wei proposed a "resource consumption model" from the perspective of individual resources, describing that stress consumes individual resources such as self-control and social support, leading to Internet addiction [9]. Stressful situations catalyze Internet addiction, which also affects negative emotions such as anxiety. The "limited self-control theory" believes that Internet addiction as a stressful situation will deplete the self-control resources needed for emotional regulation, thus, negative emotions such as anxiety may occur in individuals [10]. However, the relationship between stress and Internet addiction is not absolute. Sun based on the "resource conservation theory" (Hobofoll, 2002) believes that when the individual's psychological capital is abundant enough, Internet addiction caused by stress can be effectively avoided [11].

For the discussion of how stress affects Internet addiction, the accumulation of relevant research has been further extended. There are three kinds of research on its mechanism at present -- mediating model, moderating model and cyclic model.

\subsection{The Mediating Model}

The mediating model believes that the influence of stress on Internet addiction is achieved through a series of mediation effects [9]. Existing empirical studies have found that there are intermediary effects in the relationship between stress and Internet addiction, such as anxiety, psychological capital, basic psychological satisfaction, resources, and time management.
Researchers have found that stress can affect Internet addiction through the mediating effect of anxiety. Wang selected 1,400 undergraduates from three colleges and universities in Jingzhou City as subjects and found that anxiety plays a completely intermediary role between learning stress and Internet addiction [12]. Ye examined the relationship between the three, eventually found that anxiety plays a chain mediating role between stress and Internet addiction of college students [6]. Gao believes that severe Internet addiction can cause individuals to be disconnected from reality, which will inevitably cause them to develop anxiety, the deeper the degree of Internet addiction, the higher the level of anxiety [10].

Stress can affect Internet addiction through the intermediary role of psychological capital. Zhang put forward the interactive system model of the mechanism of Internet addiction among teenagers, namely Internet addiction behavior is the interaction of psychological, physiological and social factors, and adverse family environment and stressful events in life will reduce their self-esteem [13]. However, this does not mean that teenagers' Internet addiction is consistent with this process. Sun and other students in a higher vocational college in Guangdong Province, 266 subjects, found that psychological capital (self-efficacy, optimism, resilience, hope) in the relationship between stress and Internet addiction played a part of the intermediary role. Stress is uncontrollable and unpredictable, but individuals with high levels of psychological capital can better cope with stress and avoid negative and avoiding emotions and behaviors.

Time management mainly includes three dimensions: time value, time monitoring view and time efficacy. The research of Ren found that the higher the level of individual time relationship, the lower the level of anxiety [14]. In a survey of 610 students from key colleges and universities in Hunan Province, Zhu found that the time management tendency of college students is part of the mediating role between stress and Internet addiction [15]. In the case that individuals cannot control their own time well in the face of stress, it is easy to turn the Internet into a refuge in real life [13].

The basic psychological need is satisfied to play an intermediary role in the use of tobacco and alcohol, drug use and many other problematic uses [16]. According to the Use-and-Satisfaction Theory, people's basic psychological needs play a vital role in the healthy development of individuals. If the basic psychological needs cannot be satisfied in the external environment of reality, individuals will turn to the network to seek. Tian took 617 colleges and universities students from a university in Hunan as the data collection object, and found that basic psychological needs (autonomy needs, competence needs, relationship needs) play a partial mediating role in the relationship between the stressful 
life events faced by college students and online game addiction [5].

Stress can also affect Internet addiction through the mediation of resources. According to the analysis results of 829 valid questionnaires from a college/university in Wuhan, stress will consume individual resources (such as self-esteem, self-control, gratitude) and social resources (such as social support, peer relationship, teacher-student relationship), thus induce the adaptive problem of Internet addiction. This also reminds us that the "virtual resources" provided by the network are different from the social resources and individual resources in the usual classification, and may have a negative impact on individuals [9].

For existing research, the research on the intermediary variables of the relationship between stress and Internet addiction is more comprehensive, which can be roughly divided into two types: the individual characteristic variables of teenagers and the growth environment characteristic variables. From a quantitative point of view, however, few related studies involving environmental characteristic variables, thus more confirmation is needed on the way to create an external environment to alleviate teenagers' Internet addiction.

\subsection{The Moderating Model}

The relationship between stress and Internet addiction will be affected by the third variable (modulator variable). At present, existing studies have found the moderating effect of comprehension of social support, social support, and future time insight $[5,17,18]$.

Wei et al. used 564 university students as subjects to analyze the mediating role of social support between stress and Internet addiction and found that good family function can alleviate the effect of alienation on Internet addiction, but the moderating effect of friend support is not significant. Specifically, for teenagers with a higher tendency for Internet addiction, the role of parental support should be emphasized. higher parental support helps them cope better with stress, and the likelihood of Internet addiction will also be lower [17]

Tian took 617 colleges and university students from a college/university in Hunan as the subjects of questionnaire survey and data collection based on the stress-buffer model and the "timely help" model and found that comprehension of social support as a protective factor is between stressful life events and Internet addiction tendencies play a regulatory role [5]. Therefore, understanding that social support, as a protective factor, can buffer the negative impact of stressful life events on individuals and help them cope with difficulties steadily.

Future Time Perspective is an important indicator that reflects college students' future planning and evaluation.
Through a questionnaire survey of 530 college and university students, Zhang found that future time insight played a moderating role between stress perception and Internet addiction [18]. When the individual's time perception is weak, it will lead to greater perceived stress and easier to become addicted to the Internet.

Compared with mediating variables, most of the existing studies combined the mediation model (moderated mediation effect) to explore, but the investigation of moderating variables is relatively lacking. As an important protective factor, social support plays a buffering role when teenagers face stress [5], and its mechanism and effects are widely discussed in the research of stress and Internet addiction modulators.

\subsection{The Cyclic Model}

Stress and Internet addiction with the further study, the direct effect between them is very significant, there may be a bi-directional causal relationship between stress and Internet addiction. Therefore, the researchers integrated the one-way causal relationship between stress and Internet addiction and proposed a cyclic effect model involving intermediary latent variables. $\mathrm{Hu}$ conducted a questionnaire survey on 140 students in a domestic university, and found that there is a cyclic effect model based on anxiety as an intermediary variable between stress and Internet addiction [19], this has a certain positive effect on relieving stress and Internet addiction of college students.

\section{PREVENTION AND TREATMENT}

\subsection{Prevention}

If the internet addiction of teenagers caused by stress is fundamentally eliminated, teenagers themselves need to deal with the stress appropriately and reasonably, and correctly deal with the way of coping with the source of stress. From the perspective of Sun's research, stressors are widely present in all aspects, those that exist in themselves are called internal stressors, as well as exist in the environment are called external stressors [20]. According to ecological systems theory [34], from the perspective of the formation of stressors, teenagers have to deal with three main systems related to them: social environment system, physiological system and psychological system. Among them, the social environment mainly includes schools, native families and society. Physiology refers to the self-regulation and defense system of biological organisms. Hans Sherley's adaptation syndrome and genetic theory believe that living organisms have an innate drive to maintain a state of equilibrium throughout life. Diseases and overwork, etc., could disrupt this stable state. The mental system refers to the internal cognitive schema and consciousness system of the individual. These three systems are 
interpenetrated, interconnected, and contained with each other, and the various stress sources they induce will form a unique stress system to act on individuals in a specific way and structure. Teenagers in the face of stress from social, physiological and psychological triple, tend to seek other ways to ease the stress, immersed in the virtual world, self-satisfaction, is obtained by game upgrades to promote release without consequences against the instinctive behavior, and most addiction teenagers lack checking motive.

Here are three ways to deal with stressors: 1 . Improve self-efficacy. Self-efficacy refers to the ability to judgment and belief that an individual has about whether he can complete a task or work behavior, it is the ability perception determined by the situation [21]. Based on the research of Bandura and others, it can be concluded that the self-efficacy functions include: first, it affects the individual's choice in the face of difficulties. Second, it affects people's persistence and effort in activities. Laura L Paglis believes that people with high performance will be more confident [35]. Teenagers' self-efficacy is positively correlated with their ability to deal with problems actively and effectively. Meanwhile, selfefficacy can play the mediating roles of self-efficacy in regulating motivation and learning stress [36]. In my opinion, teenagers can improve their self-efficacy and relieve stress in the following aspects. One side is to improve self-efficacy through positive self-suggestion. Psychologist Williams believes: "No matter what insights, plans, and goals, as long as you think repeatedly with strong beliefs and expectations, it will inevitably be placed in the subconscious, and it must be in your subconscious mind as a source of positive action." Psychophysiologist Pavlov believes that "hint is the simplest and most typical conditioned reflex of mankind." When you get up or before going to bed, tell yourself silently that I will be able to deal with difficult problems with ease and I can certainly reduce psychological stress through self-motivation. Another side is to improve selfefficacy through the power of role models. The third side is by summing up the experience and lessons of past successes or failures. By recalling and summarizing some challenging things that I experienced and achieved the desired effect through my efforts, encourage yourself to overcome the difficulties in front of you. 2. Understand psychological stress correctly. According to the spring effect of stress, the greater the stress, the higher the work efficiency, but when the stress reaches a critical point, the greater the stress, the lower the work efficiency [22]. When psychological stress occurs, try to adopt a positive way to deal with the stress, convert stress into power, learn to self-regulate, reduce the physical and mental damage caused by stress. 3. Regulate stress from the physiological level. Muscles are often in a state of tension under stress. If calcium is not replenished promptly, it will react with lactic acid produced by the body, leading to fatigue and anxiety.

\subsection{Western Medicine Treatment}

Zhou et al. based on vixen-based morphometry (VBM) found that teenagers with Internet addiction often have more decision-making, behavioral and emotional problems [37]. According to research by Koo and Jeong, Internet addiction may lead to functional changes in the developing brain of teenagers. Ahmadi et al. found that the use of antipsychotic drugs can relieve the emotional symptoms of addicts [38]. Therefore, the existing therapies mostly use drug therapy combined with psychotherapy. Drug therapy mainly includes antidepressants (such as doxepin, imipramine, amitriptyline) and anti-anxiety agents (such as diazepam, Librium, nitrazepam), and scopolamine is also advocated [23]. If antidepressant or anti-anxiety drugs are used alone to treat Internet addiction, it will produce a series of adverse reactions, if the curative effect is not obvious, the side effects are large, it will be easy to relapse, and even lead to drug addiction. Han's research shows that sustained release can alter cravings and brain activity in a possible way similar to drug abuse [39]. Although the therapeutic effect of adjuvant drug therapy is significant, the toxicity of drugs will affect the physiological function and quality of life of patients. Most cognitive behavior therapy (cognitive behavior therapy) tends to use aversion therapy. If the degree is too heavy or the scale is too large to exceed the standard, it is easy to develop into a disguised form of sadism, which is counterproductive and causes symptoms.

To discuss the combination of traditional Chinese and western medicine treatment, the effect is significant and side effects are few. From the source of treatment, drug addiction is eliminated, and withdrawal reactions are effectively controlled. The mechanism of acupuncture therapy has been clear to block and inhibit the physiological basis of addictive behavior, that is, by stimulating the nervous system to release opioids, blocking the common pathway of addiction. Combining drug therapy can reduce the dosage and side effects. For the psychological problems of patients with IAD, appropriate cognitive behavioral therapy can be used to stimulate and train the brain's thinking form to eventually become the patient's spontaneous or habitual behavior, with the help of parents, new positive cognition and behavior should be established to improve the family environment and the psychological state of patients from both internal and external factors [23]. Refer to the description of the characteristics of addiction and the observation of clinical practice in the identification points of Internet addiction developed by British psychologist Griffiths, Li's research summarized the characteristics of addictive behavior including increased tolerance, withdrawal response, and compulsive use of the Internet, avoidance and avoidance, emotional behavioral responses and repetitiveness $[24,40]$. 
In the treatment of Internet addiction, the "treatment trilogy" can be used: first, according to the patient's situation and willingness, two methods, compulsory or gradual, are adopted to make the patient quit Internet addiction in behavior, namely quit the addiction. Then, psychological therapies based on behavioral therapy and cognitive therapy, and drug therapy, when necessary, gradually reduce the symptoms, until finally quit the psychological addiction, namely quit heart addiction, to achieve preliminary psychological rehabilitation. Reconstruct the personality severely affected by Internet addiction based on the patient's personality characteristics, psychological needs, and other specific conditions, and provide appropriate psychological counseling [25]. In addition, social factors need to be considered during treatment. Based on the stressor theory mentioned above, an important factor of the social environment - family and school should cooperate with treatment. Parents of teenager patients with family disabilities are encouraged to participate; schools should strengthen communication between teenager patients with problems and teachers, and the campus environment should be properly repaired.

\subsection{Traditional Chinese Medicine Treatment}

Internet addiction is a unique result of the Internet era, so traditional Chinese medicine lacks discussions on IAD. According to its symptoms, it has been classified as an emotional disease in Chinese medicine in recent years. It should be related to the heart, liver, spleen, and kidney. But the current focus of clinicians is different [26]. From the perspective of traditional Chinese medicine, IAD is classified into the category of emotional diseases based on symptoms, similar to "hepatic depression", "internal dry", etc., including the increased desire for online games and depression, mood disorders, irritability, irritability, abdominal distention, loss of appetite [27]. Therefore, it is feasible to treat IAD patients dialectically from the perspective of emotional adjustment. The main idea of "Emotion Dialectics" is a dialectical thinking process that takes the five viscera as the center, takes the physical emotional changes as the representation, and connects the emotional activities of the human body with the viscera relationship [24]. Liu and others put forward the corresponding treatment principles: In the early stage, it was mainly expected liver qi to relieve depression, qi regulating and blood activating; the late period is focused on adjusting nourishing viscera, especially spleen and heart. At present, there are mainly three kinds of TCM methods for IAD-Chinese medicine dialectical treatment, acupuncture therapy, acupuncture therapy and other intervention methods. According to clinical manifestations, Pang divided IAD into three types: liveryang upper disturbance syndrome, heart and spleen deficiency syndrome, and phlegm-fire internal disturbance syndrome. According to the dialectical results, different traditional Chinese medicine decoctions were used for treatment at the same time as psychological and behavioral therapy. Significant efficacy [28]. However, there is still a lack of a standardized program for the dialectical treatment of IAD. According to Zhou's research, acupuncture and moxibustion treatment of Internet addiction syndrome are mainly aimed at regulating the patient's qi and blood to restore the normal function of the soul, stimulate righteousness, and regulate the soul [29]. Acupuncture plus ear stress combined with psychological and drug treatment of Internet addiction syndrome can increase the basic cure rate and total effective rate of Internet addiction syndrome, and significantly improved the patient's mental symptoms. However, the study still has limitations, such as the conclusion of whether there is a correlation between the age, the severity of the disease and the efficacy of Internet addiction patients is not clear. Lv's emotional therapy, traditional Chinese medicine treatment, etc., combined with acupuncture therapy can achieve better results [30,31]. Traditional Chinese medicine characteristic therapy has promoted the treatment and research of this disease to a great extent, and has no obvious side effects and wide applicability. The previous traditional Chinese medicine treatment of IAD paid less attention to the physiological system, one of the sources of stress, while traditional Chinese medicine advocates the unity of body and mind. Psychosomatic medicine is an important part of contemporary preventive medicine, with the development of medicine and the expansion of the scope of disease management, the influence on the development of diseases has been gradually paid attention by scholars [32]. Stress will bring about physiological reactions and will produce psychological changes under the influence of physiology. The use of physiologic adjuvant therapy is suggested in future studies to better induce the perception of stress.

\section{CONCLUSION}

To conclude, stress and teenagers' Internet addiction are significantly positively correlated; stress can not only directly affect teenagers' Internet addiction, but also indirectly affect Internet addiction through the adjustment or mediation of anxiety, psychological needs, resources, and other related factors; the Internet addiction caused by stress can be treated and prevented through effective coping with stressors and integrated traditional Chinese and western medicine.

However, there are few empirical studies on the influence mechanism and environmental factors of the cyclic model and the moderating effect model alone. It is of great significance to study the relationship between stress and teenager Internet addiction in the future and explore the moderating effect of mediating variables. At the same time, it can provide a scientific and effective basis for the treatment and prevention of teenagers' Internet addiction. 


\section{REFERENCES}

[1] Gao Yuanyuan and Kong Weimin. Review on the research progress of Internet addiction $[\mathrm{J}]$. Chinese Journal of School Doctor, 2007, 021 (003): 355-358

[2] Lei Li and Li Hongli. Time perspective of teenagers, and the relationship between interpersonal involvement and Internet use [J]. Acta Psychologica Sinica, 2004, 36 (003): 335-339

[3] Yang Bo and Qin Qiwen. Biopsychosocial model of addiction $[\mathrm{J}]$. Journal of Psychological Science, 2005 (01): 32-35

[4] Zhang Xian, Wei Hua and Ding Qian. The impact of stress on online game addiction for male college students: the mediating role of self-control [J]. Studies of Psychology and Behavior, 2019, 017 (005): 713-718

[5] Tian Hui. The relationship between stressful life events and online game addiction of college students: a regulated intermediary model [D]. Hunan Normal University

[6] Ye Baojuan and Zheng Qing. Influence mechanism of stress on college students' Internet addiction [J]. Journal of Psychological Science, 2016 (3): 621-627

[7] Mei Songli, Chai Jingxin and Guo Jinhua. Teenagers' subjective happiness and Internet addiction: the mediating role of self-esteem and self-control [J]. Psychological Development and Education, 2015 (05): 603-609

[8] Tian Yu, Bian Yulong, Han Piguo, et al. The influence of shyness on Internet addiction: the mediating role of immersion tendency and Internet non-adaptive cognition [J]. Chinese Journal of Special Education, 2015, 000 (012): 83-89

[9] Wei Hua. The influence of stress on Internet addiction and its mechanism. Central China Normal University, 2014

[10] Gao Hao. The relationship between high school students' Internet addiction and stress [D]. Mudanjiang Normal University

[11] Sun Yi, Liao Wenna and Huang Liangyi. The influence of stress and psychological capital on college students' Internet addiction [J]. Journal of Guangdong Radio and Television University, 2017, 026 (006): 87-91

[12] Wang Zhengyi. Study on the relationship between college students' stressful life events, anxiety and Internet addiction. Yangtze University, 2020

[13] Zhang Kuo, Lin Jing and Fu Lifei. Interactive system model of teenagers' Internet addiction mechanism
[J]. Psychological research, 2009, 002 (002): 60-64

[14] Ren Zegang, Liu Chunxiang, He Ke, et al. Study on the relationship between college students' time management tendency, achievement motivation and anxiety [J]. Journal of Guizhou Normal University(Social Sciences), 2006 (1): 125-129

[15] Zhu Chuanwen. Study on the relationship between college students' stress, time management tendency and Internet addiction [D]. Central South University

[16] Chen Yanlei, ye Baojuan, Hu Zhujing, et al. Tobacco and alcohol use, stressful life events and gratitude attitude of middle school students [J]. Chinese Mental Health Journal, 2012, 26 (010): 796-800

[17] Wei Hua, Zhou Zongkui, Zhang Yongxin, et al. The relationship between stress and Internet addiction: the regulatory role of support from family and friend [J]. Studies of Psychology and Behavior, 2018, v.16 (02): 124-129

[18] Zhang Ye, Liu Qinxue, long Zhou, et al. The relationship between college students' trait anxiety and Internet addiction: a regulated intermediary model [J]. Psychological Development and Education, 2016, 32 (006): 745-752

[19] Hu Junhang, and Tong Hengqing. Analysis on the intermediary circular effect between college students' stress and Internet addiction [J]. Journal of Campus Life \& Mental Health, 2020 (3): 179-181

[20] Sun Tao. Study on the relationship between stress source and coping styles of senior school students [D]. Inner Mongolia Normal University

[21] Huo Tuanying. Investigation on the psychological stress of grass-roots party and government cadres and adjusting countermeasures [J]. Leadership Science, 2010

[22] Wei Shuangfeng. The relationship between college sudents' loneliness, campus stress and coping style [J]. Journal of Liaoning Medical College:Social Science Edition, 2012, 10 (4): 53-55

[23] Liu Yangyang, Su Peizhu, Zhang Guifeng, et al. Discussion on clinical ideas of TCM in the treatment of Internet addiction [J]. Modern Medicine Journal of China, 2010, 012 (005): 119-121

[24] Li Geng. Cognitive behavioral therapy for teenagers with Internet addiction [D]. Ningxia Medical University, 2009

[25] Yang Rong, Shao Zhi and Zheng Yong. Comprehensive intervention for middle school students with Internet addiction [J]. Chinese Mental Health Journal, 2005, 19 (007): 457-459

[26] Dai Yu, Zhu Tianmin, Wen Chao, et al. Study 
progress in the treatment of Internet addiction syndrome $[\mathrm{J}]$. Journal of Community Medicine, 2017, 015 (001): 83-86

[27] Chen Yuhui. Discussion on the construction of emotional discipline of traditional Chinese medicine and the building of clinical prevention and treatment framework of emotional diseases [D]. Guangzhou University of Chinese Medicine, 2005

[28] Pang Yin. Treatment on juveniles with Internet addiction syndrome by integrated traditional Chinese and Western Medicine [J]. Modern Journal of Integrated Traditional Chinese and Western Medicine, 2009 (4)

[29] Zhou Dezhao. Clinical study on acupuncture plus ear stress in the treatment of Internet addiction syndrome [D]. Guangzhou University of Chinese Medicine, 2011

[30] LV Lei. Psychological system of Chinese Medicine in Huang Di Nei Jing Su Wen [J]. Chinese Journal of Tissue Engineering Research, 2005, 9 (024): 192193

[31] Huang Shile, Zhang Chunxia, Yuan Mei, et al. Relationship between Internet addiction and mind and discussion on syndrome differentiation and treatment of Chinese medicine [J]. Journal of New Chinese Medicine, 2006, 038 (009): 8-9

[32] Zhang Le, Zhang Jing and Lin Hong. Application of comfort management model of TCM body-mind integration theory in oncology $[\mathrm{J}]$. Journal of TCM management, 2020, V.28; No.330(23):163-164.

[33] Davis R A. A cognitive-behavioral model of pathological Internet use[J]. Computers in Human Behavior, 2001, 17(2):187-195.

[34] Bronfenbrenner U. Lewinian Space and Ecological Substance[J]. Journal of Social Issues, 1977, 33(4):199-212.

[35] Paglis L L, Green S G. Leadership self-efficacy and managers' motivation for leading change[J]. Journal of Organizational Behavior, 2002, 23.

[36] Wu H, Li S, Zheng J, et al. Medical students' motivation and academic performance: the mediating roles of self-efficacy and learning engagement $[\mathrm{J}]$.

[37] Zhou Y, Lin F C, Du Y S, et al. Gray matter abnormalities in Internet addiction: A voxel-based morphometry study[J]. European Journal of Radiology, 2011, 79(1):92-95.

[38] Ahmadi J, Amiri A, Ghanizadeh A, et al. Prevalence of Addiction to the Internet, Computer Games, DVD, and Video and Its Relationship to Anxiety and
Depression in a Sample of Iranian High School Students[J]. Iranian Journal of Psychiatry and Behavioral Sciences, 2014, 8(2):75-80.

[39] Han D H, Hwang J W , Renshaw P F . Bupropion sustained release treatment decreases craving for video games and cue-induced brain activity in patients with Internet video game addiction.[J]. Experimental \& Clinical Psychopharmacology, 2010, 18(4):297.

[40] Griffiths, Mark. Does Internet and Computer ।"Addiction\" Exist? Some Case Study Evidence[J]. Cyberpsychology \& Behavior, 2000, 3(2):211-218. 